\title{
CROMOBLASTOMICOSE NO RIO GRANDE DO SUL: RELATO DE 12 CASOS
}

\author{
Shirley M.W. Matte, Jorge O. Lopes, Ivanir S. Melo, \\ Luís Eduardo R. Espadim e Marcelo Souza Pinto
}

\begin{abstract}
São relatados 12 casos de cromoblastomicose diagnosticados no interior do Rio Grande do Sul, no período 1988-1995. Os aspectos clínicos e evolutivos são analisados e comparados com a literatura. O único agente isolado foi Fonsecaea pedrosoi.
\end{abstract}

Palavras-chaves: Cromoblastomicose. Fonsecaea pedrosoi. Rio Grande do Sul.

A cromoblastomicose é micose subcutânea de aspecto polimórfico, acometendo a pele e o tecido celular subcutâneo. Caracteriza-se pelo surgimento de lesão pápulo-descamativa, que evolui lenta e progressivamente para lesões cutâneas variadas, com sintomatologia discreta17 e a presença de células escleróticas ou muriformes no exame microscópico do pus ou tecidos7. O principal agente é a Fonsecaea pedrosoi. A doença é também causada por $F$. compacta, Cladosporium carrionii, Rhinocladiella aquaspersa e Phialophora verrucosa 5 . Mais recentemente têm sido relatados casos por Exophiala jeanselmei e E. castellanii12, e por E. spinifera8. Os agentes são encontrados na natureza, e isolados do solo, ar e vegetais 41317. A penetração do fungo no organismo ocorre geralmente por implantação traumática de material orgânico contaminado pelos conídeos e micélio destes fungos 34 13. É doença de distribuição cosmopolita, com maior incidência nas regiões tropicais e subtropicais de clima quente e úmido da América Latina, África e Ásia. No Brasil, o Rio Grande do Sul, São Paulo, Rio de Janeiro, Minas Gerais, e estados da região Amazônica são áreas endêmicas 15.

Neste trabalho, analisamos os aspectos clínicos e evolutivos de 12 casos de cromoblastomicose diagnosticados no Serviço de Dermatologia do Hospital Universitário de Santa Maria (SDHUSM).

Serviços de Dermatologia e Micologia, Hospital Universitário, Universidade Federal de Santa Maria.

Endereço para correspondência: Dr. Jorge O. Lopes. Dept ${ }^{\circ}$ de Microbiologia e Parasitologia/UFSM, Faixa de Camobí, Km 09, 97119-900, Santa Maria, RS, Brasil. Fax:(055) 226-1616, ramal 8742.

Recebido para publicação em 25/09/96.

\section{MATERIAL E MÉTODOS}

Foram revisados os prontuários médicos dos pacientes com diagnóstico de cromoblastomicose atendidos no ambulatório do SDHUSM no período de janeiro de 1988 a janeiro de 1995. Dados pessoais, características e tempo de evolução da lesão cutânea, terapêutica utilizada e resultado clínico-laboratorial do tratamento, foram analisados. O diagnóstico de cromoblastomicose foi obtido pelo exame micológico direto em potassa (KOH $10 \%)$, cultivo em Mycosel (BBL) a $25^{\circ} \mathrm{C}$, e exame histopatológico.

Somente cinco pacientes tiveram acompanhamento clínico, e os critérios de cura foram os mesmos adotados por Queiroz-Telles e cols11: completa cicatrização (cura) de todas as lesões; ausência de parasitas no exame direto e cultivos negativos; persistência desses achados em três biópsias consecutivas, de diferentes locais da lesão.

\section{RESULTADOS}

Os dados dos 12 casos são mostrados na Tabela 1. A média de idade foi de 51 anos, variando de 34 a 68 anos, e a relação homem/mulher foi de 11/1. Dez pacientes eram agricultores e dois viviam na zona urbana. Todos eram brancos, naturais do Rio Grande do Sul, e procedentes de cidades próximas à Santa Maria. Sete pacientes referiam história de traumatismo prévio no local da lesão cutânea e cinco apresentavam lesão há pelo menos 10 anos. Os membros inferiores, particularmente as pernas e os pés, estavam acometidos em nove pacientes. Quanto aos aspectos morfológicos das lesões, a forma verrucosa teve nítida prevalência, sendo geralmente lesão única. Sangramento ( 4 casos) e dor (3 casos) foram os sintomas mais referidos na consulta inicial. 


\begin{tabular}{|c|c|c|c|c|c|}
\hline Caso & Evolução & Local & Lesão & Terapêutica* & Resposta $^{* * *}$ \\
\hline 1 & 8 meses & tornozelo & nodular & FC & cura \\
\hline 2 & 20 anos & pé & verrucosa & ITC/CL & cura \\
\hline 3 & 3 anos & periumbilical & verrucosa & $\mathrm{RC} / \mathrm{FC}$ & cura \\
\hline 4 & 4 anos & tornozelo & verrucosa & $\mathrm{AB} / \mathrm{FC} / \mathrm{CL}$ & SA \\
\hline 5 & 10 anos & tornozelo & verrucosa & $\mathrm{AB} / \mathrm{FC} / \mathrm{CL}$ & SA \\
\hline 6 & 5 anos & pé & verrucosa & NR & SA \\
\hline 7 & 17 anos & tornozelo & verrucosa & $\mathrm{ITC} / \mathrm{FC} / \mathrm{CL} / \mathrm{EC}$ & SA \\
\hline 8 & 10 anos & punho & verrucosa & NR & SA \\
\hline 9 & 30 anos & pé & verrucosa & ITC/CL & SA \\
\hline 10 & 5 anos & pé & verrucosa & $\mathrm{RC} / \mathrm{CL}$ & SA \\
\hline 11 & 4 anos & punho & verrucosa & $\mathrm{RC}$ & SA \\
\hline 12 & 4 meses & pé & verrucosa & RC/ITC & cura \\
\hline
\end{tabular}

O diagnóstico da doença foi possível em todos os casos, através de exame micológico direto e cultivo, e do exame histopatológico. Os cultivos permitiram a identificacação da $F$. pedrosoi como o único agente causal.

O tratamento administrado foi variável, com a utilização de anfotericina B, 5-fluorocitosina e itraconazole, isolados ou associados. Ressecção cirúrgica e calor também foram utilizados. Dois pacientes não se submeteram ao tratamento após diagnóstico.

Mais da metade dos pacientes registrados não retornaram para controle da doença em alguma fase do tratamento. Em quatro casos observou-se cura clínica, micológica e histopatológica. A regressão clínica parcial das lesões foi observada em um caso.

\section{DISCUSSÃO}

Os agricultores do sexo masculino, como nos nossos casos, são as grandes vítimas da cromoblastomicose. Resultado semelhante foi observado por Silva e cols15 no estado do Maranhão, e Cucé em São Pauló 4 , concordando assim com Lacaz, quando este se refere que os trabalhadores rurais do sexo masculino pagam o maior tributo à infecção5.

Todos os pacientes eram brancos, o que pode ser explicado pela predominância étnica de origem européia em nosso meio, pois tal fato não se observa em outras regiões cuja composição etnica é diferente.

Como na série de 93 casos relatados por Bopp e Bernardi ${ }^{1}$, e nos 73 casos estudados por Londero e Ramos 6 , em nosso estudo também houve uma distribuição geográfica peculiar no que se refere à procedência dos pacientes, com todos oriundos da metade norte do Rio
Grande do Sul, em regiões localizadas acima do paralelo $30^{\circ}$ Sul. Bopp e Bernardi correlacionaram este achado com a existência de fatores climáticos, revestimento e composição do solo, que favoreceria a vegetação dos agentes da cromoblastomicose na natureza. Londero e Ramos destacam também que nestas regiões predomina a pequena propriedade rural, sendo hábito do agricultor trabalhar diretamente na terra, descalço e, usualmente, de calças arregaçadas.

Houve uma diminuição no número de casos/ano de cromoblastomicose: 2,5 casos/ano na série de Londero e Ramos 6 , e 1,5 casos/ano na presente série.

A localização predominante das lesões em membros inferiores e história de trauma local prévio, referido por $58,3 \%$ dos pacientes, correlaciona-se diretamente com o tipo de atividade desempenhada pelos mesmos e reforça a hipótese da implantação traumática do fungo nos tecidos para produzir esta infecção. Esses achados concordam com a maioria dos autores 131517 .

Em nossos casos a maioria das lesões iniciaram como pápulas ou nódulos, únicas, de aspecto verrucoso. Complicações tardias como ulceração, fibrose, diminiuição da mobilidade, bloqueio linfático causando linfedema e elefantíase, e carcinoma de células escamosas 131517 , não foram significativas. O achado dos elementos muriformes globosos, de paredes fuliginosas e espessas, septados em dois planos, característicos dos agentes da cromoblastomicose através do exame micológico direto em todos os casos, demonstra a facilidade diagnóstica desta micose, podendo ser realizado com poucos recursos laboratoriais. Quanto à natureza dos agentes etiológicos 
isolados através de cultivo, houve um predomínio absoluto da $F$. pedroso $i$, responsável pela maioria dos casos da doença no Rio Grande do Sul e no Brasil4 617.

A cromoblastomicose ainda constitui um desafio terapêutico, destacando-se atualmente a exerése cirúrgica das lesões pequenas2 39 16, e o tratamento com itraconazol ou anfotericina B11 17, associados a 5-fluorocitosina 10 nos casos em que a cirurgia não é factivel. Porém, nenhum tratamento tem sido associado com resultados consistentes o suficente para ser considerado de escolha3. Diversos esquemas terapêuticos foram utilizados em nossos pacientes. Entretanto, devido ao alto custo, condições sócio-econômicas baixas e longo tempo de tratamento, mais de $50 \%$ dos pacientes não retornaram ao SDHUSM para controle da doença.

\section{SUMMARY}

Twelve cases of chromoblastomycosis diagnosed in Rio Grande do Sul during 1988-1995 are reported. The clinical aspects are analyzed and compared with the literature. Fonsecaea pedrosoi was the only microorganism isolated.

Key-words: Chromoblastomycosis. Fonsecaea pedrosoi. Rio Grande do Sul

\section{REFERÊNCIAS BIBLIOGRÁFICAS}

1. Bopp C, Bernardi C. Geopatologia da cromoblastomicose no Rio Grande do Sul. Medicina Cutanea Ibero-Latino-Americana 7:1-8, 1973.

2. Castro LGM. Mecanismo de cura da cromomicose pela criocirurgia com nitrogênio líquido. Anais Brasileiros de Dermatologia 63:297-300, 1989.

3. Castro LGM. Chromomycosis. A therapeutic chalange (corresp). Clinical Infectious Diseases 15:553, 1992 .

4. Cucé LC, Salebian A, Gatti CF, Sampaio SAP. Cromomicosis: Estudio de 37 casos. Revista Argentina de Dermatologia 64:1-15, 1983.

5. Lacaz CS, Porto E, Martins JEC. Cromoblastomicose. In: Micologia médica - fungos, actinomicetos $\mathrm{e}$ algas de interesse médico. Editora Sarvier, São Paulo, 1984.

6. Londero AT, Ramos CD. Cromoblastomicose no interior do Estado do Rio Grande do Sul. Anais Brasileiros de Dermatologia 64:155-58,1989.

7. Odds FC, Arai T, Di Salvo AF, Evans EG, Hay RJ, Ranothawa HS, Rinaldi MG,Walsh TJ. Nomenclature of fungal diseases: a report and recommendations from a sub-committee of the International Society for Human and Animal Mycology (ISHAM). Journal of Medical and Veterinary Mycology 30:1$10,1992$.

8. Padhye AA, Hampton AA, Hampton NT, Hutton NW, Prevost-Smith E, Davis MS. Chromoblastomycosis caused by Exophiala spinifera. Clinical Infectious Diseases 22:331335, 1996.

9. Pimentel, ERA, Castro LGM, Cucé LC, Sampaio SAP. Treatment of chromomycosis by cryosurgery with liquid nitrogen: an report of eleven cases. Journal of Dermatology and Surgery Oncology 15:72-77, 1989.

10. Pradinaud R. Treatment of chromoblastomycosis (corresp). Journal of American Academy of Dermatology 25:869, 1991.

11. Queiroz-Telles F, Purim KF, Fillus JN, Bordignon GF, Lameira RP, Van Cutsem J, Cauwenbergh G. Itraconazole in the treatment of chromoblastomycosis due to Fonsecaea pedrosoi. International Journal of Dermatology 31:805-812, 1992.

12. Queiroz-Telles F, Wanke B, Monteiro PCF, Fernandes-Bordignon GP, Pinheiro RL, Nishino L. Chromoblastomycosis caused by different species of Exophiala. In: Resumo do VII Congresso Brasileiro de Infectologia, Porto Alegre, p.113, 1994.

13. Rubin HA, Bruce S, Rosen S, McBride ME. Evidence for percutaneous inoculation as the mode of transmission for chromoblastomycosis. Journal of American Academy of Dermatology 25:951-954, 1992.

14. Sampaio SAP. A síndrome verrucosa. Anais Brasileiros de Dermatologia 60:247-252, 1985.

15. Silva ACCM, Neto AS, Galvão CES, Marques SG, Saldanha ACR, Silva, CMP, Fischman O, Silva RR, Costa MRSR, Costa JML. Cromoblastomicose produzida por Fonsecaea pedrosoi no Estado do Maranhão I - Aspectos clínicos, epidemiológicos e evolutivos. Revista da Sociedade Brasileira de Medicina Tropical 25:37-44, 1992.

16. Sittart JAS, Valente NYS. Tratamento da cromomiose pela criocirurgia com nitrogênio líquido. Medicina Cutânea Ibero-Latino-Americana 14:227-232, 1986.

17. Tuffanilli L, Milburn PB. Treatment of chromoblastomycosis. Journal of American Academy of Dermatology 23:728-32, 1990. 